\title{
Sobre um novo método de inversão de matrizes de Vandermonde para solução de recorrências lineareas homogêneas de ordem superior
}

\author{
Lucas S. C. de Sá \\ Elen V. P. Spreafico
}

\section{Resumo}

Este artigo apresenta um estudo sobre a resolução de recorrências lineares homogêneas de ordem superiores cujo polinômio característico associado possui raízes simples, abordando o método tradicional e um novo método já publicado envolvendo o que advém das sequências de Fibonacci generalizadas, na obtenção direta da inversa da matriz de Vandermonde, matriz essa dada no sistema linear associado a recorrência. Apresentaremos como aplicação a resolução de problemas existentes na literatura com recorrências de ordem 2 associados, fazendo um comparativo de ambos os métodos de resolução.

Palavras-chave: Relação de Recorrências; Termo Geral; Sequências de Fibonacci Generalizadas; Aplicações.

\section{Abstract}

This article presents a study on the resolution of homogeneous linear recurrences of higher order whose associated characteristic polynomial has simple roots, addressing the traditional method and a new method already published involving results of generalized Fibonacci sequences, in obtaining the inverse of the Vandermonde matrix, associated with the recurrence. We will present as an application the solution of problems existing in the literature, associated with recurrences of order 2 , making a comparison of both methods.

Keywords: Linear Recurrence; Vandermonde Matrix; Generalized Fibonacci Sequences; General Term of Sequences.

\section{Introdução}

A palavra recursão remete ao século 19, no desenvolvimento formal de funções. Datado em 1888, a recursão teve uso por Dedekind [5] para obter funções que precisava na formalização analítica dos conceitos de número natural. Em 1923, Skolem utilizou a ideia de recursão para definir funções básicas na lógica. A ideia de sequências em que termos posteriores são deduzidos a partir dos anteriores existe desde a antiguidade, fato nótorio nos métodos de indução e aproximação.

Desde 1600 tem-se como ideia clara sequências de inteiros definidas através de recursão, as relações de recorrência de inteiros. Com o processo de formalização da linguagem matemática no fim do século 19 e começo do século 20, elas puderam ser exploradas, e ainda continuam sendo estudadas. 
Resolver uma recorrência consiste em encontrar uma função ou família de funções que dependam somente de $n$ satisfazendo a recorrência. Na literatura existem vários métodos de resolução de uma recorrência levando em conta suas características: ordem linear, não linear, homogênea e não homogênea. Os casos mais básicos são as recorrências advindas das progressões aritméticas e geométricas, essas vistas no ensino básico.

O fato mais interessante é que, dadas as condições iniciais, a resolução de uma recorrência de ordem superior recai na resolução de um sistema linear associado à matriz de Vandermonde. Diante desse fato, neste artigo retomamos o método tradicional e apresentamos um método diferente do tradicional que resolve o sistema linear através da obtenção direta dos termos da matriz inversa de Vandermonde, e, assim, resolve o sistema.

Inicialmente introduzimos as sequências de Fibonacci generalizadas e discorremos sobre ambos os métodos, tradicional e novo, este que chamaremos método BenTaher-Rachidi, para resolução de uma recorrência linear homogênea de ordem superior com polinômio característico associado com raízes simples. Em seguida são apresentadas aplicações com resolução de exemplos de recorrência linear de ordem 2 em ambos os métodos para um comparativo.

\section{Aspectos Teóricos}

Nesta seção, alguns conceitos fundamentais de relação de recorrência são apresentados, como também os métodos mais tradicionais de resolução e um novo método que não é amplamente divulgado na literatura, e que será chamado de método de BenTaher-Rachidi. Mais detalhes a respeito desses métodos podem ser encontrados nas referências [2] e [4].

Definição 1 (Sequência de Fibonacci generalizada). Dadas as constantes $a_{0}, a_{1}, \ldots, a_{r-1}$, com $a_{r-1} \neq 0$, e $V_{0}, V_{1}, \ldots, V_{r-1}$ no corpo dos números reais ou complexos, a sequência $\left(V_{n}\right)_{n \geq 0}$ ao ser representada pela relação de recorrência linear homogênea de ordem r,

$$
V_{n+1}=a_{0} V_{n}+\ldots+a_{r-1} V_{n-r+1},
$$

para $n \geq r-1$, é chamada de sequência generalizada de Fibonacci [1].

\section{Exemplo 1.}

a) A relação de recorrência de ordem 2 com coeficientes $a_{0}=1, b_{0}=1$ e $V_{0}=1, V_{1}=3$ é descrita por $V_{n+1}=V_{n}+V_{n-1}$ e é conhecida como sequência de Lucas.

b) A relação de recorrência de ordem 2 com coeficientes $a_{0}=1, b_{0}=1$ e $V_{0}=1, V_{1}=1$ é descrita por $V_{n+1}=V_{n}+V_{n-1}$ e é conhecida como sequência de Fibonacci.

Ambos os exemplos citados anteriormente são sequências generalizadas de Fibonacci. Note que apesar das duas sequências serem descritas pela mesma relação de recorrência, a constante $V_{1}$ é diferente em cada caso, gerando sequências distintas.

Definição 2 (Polinômio característico). Dada uma relação de recorrência homogênea de ordem $\mathrm{r}$, $V_{n+1}=a_{0} V_{n}+\ldots+a_{r-1} V_{n-r+1}$, o polinômio,

$$
p(z)=z^{r}-a_{0} z^{r-1}-\ldots-a_{r-2} z-a_{r-1},
$$

é chamado de polinômio característico da relação de recorrência $V_{n}$ com raízes $\lambda_{1}, \lambda_{2}, \ldots, \lambda_{s}$ e respectivas multiplicidades $m_{1}, m_{2}, \ldots, m_{s}$. 


\section{Exemplo 2.}

a) $O$ polinômio característico da relação de recorrência homogênea de ordem dois, $V_{n+1}=-V_{n}+$ $2 V_{n-1}$, é dado por $p(z)=z^{2}+z-2$ e apresenta como raízes $\lambda_{1}=-2$ e $\lambda_{2}=1$, ambos com multiplicidade um, $m_{1}=m_{2}=1$.

b) $O$ polinômio característico da relação de recorrência homogênea de ordem três, $V_{n+1}=-V_{n}+$ $5 V_{n-1}-3 V_{n-2}$, é dado por $p(z)=z^{3}+z^{2}-5 z+3$ e apresenta como raízes $\lambda_{1}=1$, com multiplicidade dois, $m_{1}=2$ e $\lambda_{2}=-3$, com multiplicidade um, $m_{2}=1$.

Note que a ordem da recorrência sempre coincide com o grau do polinômio.

\section{Métodos de resolução de uma recorrência}

Resolver uma recorrência consiste em encontrar uma função ou família de funções que dependam somente de $n$ e satisfaçam a relação de recorrência em questão.

As recorrências lineares homogêneas podem ser resolvidas por diferentes métodos. Nesse artigo, a primeira abordagem consiste em determinar as raízes do polinômio característico e suas constantes através de um sistema linear, método clássico esse frequentemente estudado nos cursos de matemática. Essa abordagem será referida como método tradicional, em comparativo com o novo método apresentado no artigo [4], nomeado aqui de método de BenTaher-Rachidi.

\subsection{Método tradicional}

Para resolver relações de recorrência linear como a apresentada em (1), é amplamente utilizada a fórmula,

$$
V_{n}=\sum_{i=1}^{s}\left(\sum_{j=0}^{m_{i}-1} \beta_{i, j} n^{j}\right) \lambda_{i}^{n},
$$

para $n \geq 0$, em que $\beta_{i, j}$ são coeficientes determinados através de um sistema linear de $r$ equações que se utiliza como condição de contorno os coeficientes $\left(V_{j}\right)_{0 \leq j \leq r-1}$, e $s$ é a quantidade de raízes distintas do polinômio característico da recorrência dada.

Exercício 1. Determine uma expressão para a relação de recorrência $V_{n+1}=-2 V_{n}+3 V_{n-1}$, sabendo que $V_{0}=1$ e $V_{1}=3$.

\section{Solução:}

O polinômio característico da recorrência será dado por $p(z)=z^{2}+2 z-3$, com $\lambda_{1}=-3$ e $\lambda_{2}=1$ e ambos com multiplicidade um, $m_{1}=m_{2}=1$.

Utilizando a fórmula 3, obtemos as equações

$$
\left\{\begin{array}{c}
V_{n}=\beta_{1,0} \lambda_{1}^{n}+\beta_{2,0} \lambda_{1}^{n} \\
V_{n}=\beta_{1,0}(1)^{n}+\beta_{2,0}(-3)^{n}
\end{array}\right.
$$

Montando o sistema linear utilizando as condições de contorno $V_{0}=1$ e $V_{1}=3$, obtemos

$$
\left\{\begin{array}{c}
\beta_{1,0}+\beta_{2,0}=1 \\
\beta_{1,0}-3 \beta_{2,0}=3
\end{array}\right.
$$


Ao resolver o sistema linear, $\beta_{1,0}=\frac{3}{2}$ e $\beta_{2,0}=-\frac{1}{2}$. Com isso, a solução da recorrência é dada pela família $V_{n}=\frac{3}{2}-\frac{(-3)^{n}}{2}$.

\subsection{Método BenTaher-Rachidi}

Note que o sistema linear a ser resolvido para determinar as constantes $\beta_{i, j}$, para os casos em que o polinômio característico da recorrência (1) apresenta apenas raízes simples, é dado por,

$$
\left\{\begin{array}{ccccccccc}
\beta_{1,0} & + & \beta_{2,0} & + & \ldots & + & \beta_{r, 0} & = & V_{0} \\
\lambda_{1} \beta_{1,0} & + & \lambda_{2} \beta_{2,0} & + & \ldots & + & \lambda_{r} \beta_{r, 0} & = & V_{1} \\
& & & & & & & \\
\lambda_{1}^{r-1} \beta_{1,0} & + & \lambda_{2}^{r-1} \beta_{2,0} & + & \ldots & + & \lambda_{r}^{r-1} \beta_{r, 0} & = & V_{r-1} .
\end{array}\right.
$$

Definição 3 (Matriz de Vandermonde). Dada uma matriz quadrada em que cada coluna (ou linha) é uma progressão geométrica onde o primeiro termo é 1, esse tipo de matriz é chamado de matriz de Vandermonde.

$$
\left[\begin{array}{cccc}
1 & 1 & \ldots & 1 \\
\alpha_{1} & \alpha_{2} & \ldots & \alpha_{m} \\
& & \vdots & \\
\alpha_{1}^{m} & \alpha_{2}^{m} & \ldots & \alpha_{m}^{m}
\end{array}\right]
$$

Com isso, o sistema linear (4) pode ser escrito como $A x=b$, em que $A$ é uma matriz de Vandermonde, $x$ é o vetor das incógnitas $\beta_{i, 0}$ e $b$ é vetor das condições de contorno da recorrência.

No artigo de BenTaher-Rachidi [4] é mostrada uma técnica para encontrar os coeficientes $\beta_{i, 0}$, e consequentemente a solução da recorrência (1), sem necessidade de recorrer à resolução de um sistema linear para os casos em que $A$ é uma matriz de Vandermonde. Com isso, a solução de uma recorrência linear homogênea cujo polinômio característico tenha apenas raízes simples é dada pela equação,

$$
V_{n}=\sum_{i=1}^{r} \frac{1}{p^{\prime}\left(\lambda_{i}\right)}\left(\sum_{p=0}^{r-1} \frac{A_{p}}{\lambda_{i}^{p+1}}\right) \lambda_{i}^{n},
$$

para $n \geq r$, onde $A_{p}=a_{r-1} V_{p}+\ldots+a_{p} V_{r-1}$.

Note que essa abordagem não requer a resolução de um sistema linear como é exigido ao utilizar o método tradicional.

Exercício 2. Determine uma expressão para a relação de recorrência $V_{n+1}=3 V_{n}-2 V_{n-1}$, sabendo que $V_{0}=1$ e $V_{1}=2$.

\section{Solução:}

O polinômio característico da recorrência será dado por $p(z)=z^{2}-3 z+2$, com $\lambda_{1}=1$ e $\lambda_{2}=2$ e ambos com multiplicidade um, $m_{1}=m_{2}=1$. Com isso é possível aplicar o método BenTaherRachidi e a solução da recorrência será dada pela fórmula (5),

$$
V_{n}=\frac{1}{p^{\prime}\left(\lambda_{1}\right)}\left(\frac{A_{0}}{\lambda_{1}^{0+1}}+\frac{A_{1}}{\lambda_{1}^{1+1}}\right) \lambda_{1}^{n}+\frac{1}{p^{\prime}\left(\lambda_{2}\right)}\left(\frac{A_{0}}{\lambda_{2}^{0+1}}+\frac{A_{1}}{\lambda_{2}^{1+1}}\right) \lambda_{2}^{n} .
$$


Precisamos determinar as contantes $A_{p}$ e $p^{\prime}\left(\lambda_{i}\right)$. De fato,

$$
\begin{gathered}
A_{0}=a_{1} V_{0}+a_{0} V_{1} \Rightarrow A_{0}=-2 \cdot 1+3 \cdot 2 \Rightarrow A_{0}=4 \\
A_{1}=a_{1} V_{1} \Rightarrow A_{1}=-2 \cdot 2 \Rightarrow A_{1}=-4 .
\end{gathered}
$$

Derivando o polinômio $p(z), p^{\prime}(z)=2 z-3$, $\operatorname{logo} p^{\prime}(1)=-1$ e $p^{\prime}(2)=1$. E, portanto, substituindo na equação obtida de (5), obtemos $V_{n}=2^{n}$.

\section{Aplicações}

Nesta seção são abordados dois problemas conhecidos que podem ser descritos por recorrência, e ambos são solucionados utilizando os dois métodos, mostrando que é possível chegar na mesma solução independentemente do método utilizado.

Problema 1 (Sequência de Fibonacci) Um homem pôs um par de coelhos num lugar cercado por todos os lados por um muro. Quantos pares de coelhos podem ser gerados a partir desse par em um ano se, supostamente, todos os meses cada par dá à luz um novo par, que é fértil a partir do segundo mês?

O problema descrito pode ser visto como apresentado na figura 1.

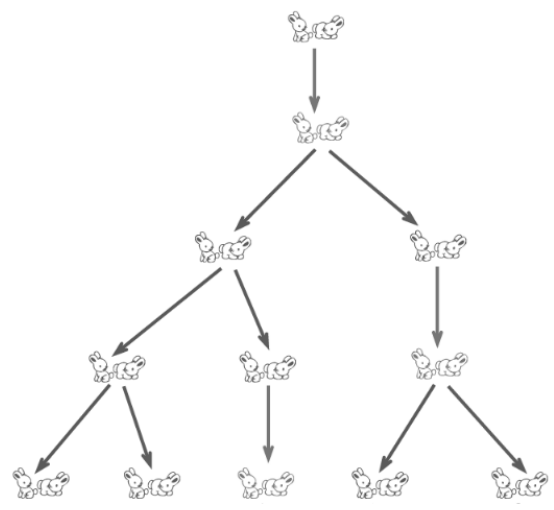

Figura 1: Representação do problema 1 ao se passar 4 meses [3].

Chamando de $F_{n}$ a quantia de pares de coelhos ao se passarem $n$ meses, pode-se afirmar que $F_{1}=1$ e $F_{2}=1$.

Para $n \geq 2$, note que o problema em questão pode ser modelado por uma relação de recorrência. No n-ésimo mês terá um total de $F_{n}$ coelhos, sendo que todos os recém-nascidos não poderão reproduzir no mês seguinte, ou seja, somente os coelhos nascidos no mês anterior, $F_{n-1}$, poderão reproduzir. 
Dessa forma, no mês seguinte o total de coelhos será igual à quantia de coelhos existentes no mês $n$ mais a quantia de coelhos que nasceram dos coelhos já vivos no mês $n-1$, sendo possível gerar a recorrência,

$$
F_{n+1}=F_{n}+F_{n-1}
$$

Assim, o problema em questão pode ser simplesmente resolvido calculados os termos da recorrência até que se encontre o número de coelhos ao se passarem doze meses, $F_{12}$.

Montando a sequência, $(1,1,2,3,5,8,13,21,34,55,89,144,233, \ldots)$, percebe-se que $F_{12}=233$, ou seja, podem ser gerados um total de 233 coelhos dentro de um ano.

Podemos generalizar a solução desse problema obtendo uma fórmula fechada para $F_{n}$ ao resolver sua relação de recorrência pelos métodos apresentados previamente.

\section{a) Método tradicional:}

O polinômio característico da expressão acima será dado por $p(z)=z^{2}-z-1$, que apresenta como raízes $\lambda_{1}=\frac{1+\sqrt{5}}{2}$ e $\lambda_{2}=\frac{1-\sqrt{5}}{2}$ e respectivas multiplicidades $m_{1}=1$ e $m_{2}=1$. Sendo assim, a relação de recorrência ao se utilizar a fórmula (3) será dada por

$$
F_{n}=\beta_{1,0} \lambda_{1}^{n}+\beta_{2,0} \lambda_{2}^{n},
$$

faltando determinar as constantes $\beta_{1,0}$ e $\beta_{2,0}$.

Resolvendo o sistema linear,

$$
\left\{\begin{array}{c}
\beta_{1,0}+\beta_{2,0}=1 \\
\lambda_{1} \beta_{1,0}+\lambda_{2} \beta_{2,0}=1
\end{array}\right.
$$

obtêm-se, $\beta_{1,0}=\frac{1}{\sqrt{5}}\left(\frac{1+\sqrt{5}}{2}\right)$ e $\beta_{2,0}=-\frac{1}{\sqrt{5}}\left(\frac{1-\sqrt{5}}{2}\right)$.

Com isso, a solução da recorrência é dada por

$$
F_{n}=\frac{1}{\sqrt{5}}\left[\left(\frac{1+\sqrt{5}}{2}\right)^{n+1}-\left(\frac{1-\sqrt{5}}{2}\right)^{n+1}\right]
$$

\section{b) Método BenTaher-Rachidi:}

Utilizando o mesmo polinômio característico que o apresentado no item $a$ ), derivando $p$ obtemos o polinômio $p^{\prime}(z)=2 z-1$ e podemos calcular $p^{\prime}\left(\lambda_{1}\right)$ e $p^{\prime}\left(\lambda_{2}\right)$.

Utilizando as condições de contorno podemos calcular $A_{1}=a_{1} V_{1}$ e $A_{2}=a_{1} V_{2}+a_{2} V_{1}$, onde $a_{0}=1$ e $a_{1}=1$, dados pelos coeficientes da relação de recorrência. Com isso, utilizando a fórmula (5), a recorrência é dada por,

$$
F_{n}=\frac{1}{p^{\prime}\left(\lambda_{1}\right)}\left(\frac{A_{0}}{\lambda_{1}^{0+1}}+\frac{A_{1}}{\lambda_{1}^{1+1}}\right) \lambda_{1}^{n}+\frac{1}{p^{\prime}\left(\lambda_{2}\right)}\left(\frac{A_{0}}{\lambda_{2}^{0+1}}+\frac{A_{1}}{\lambda_{2}^{1+1}}\right) \lambda_{2}^{n} .
$$

Substituindo os valores obtidos previamente e manipulando a equação, é possível escrever a recorrência como

$$
F_{n}=\frac{1}{\sqrt{5}}\left[\left(\frac{1+\sqrt{5}}{2}\right)^{n+1}-\left(\frac{1-\sqrt{5}}{2}\right)^{n+1}\right]
$$


obtendo o mesmo resultado que quando utilizado o método tradicional.

Problema 2 (Análise combinatória) Quantas são as sequências de $n$ termos, todos pertencentes a $\{0,1,2\}$, que não possuem dois termos consecutivos iguais a 0 ?

Note que esse problema não é facilmente solucionado utilizando apenas as ferramentas mais simples de análise combinatória, no entanto ele pode ser modelado por uma recorrência como mostrado em seguida.

Seja $S_{n+1}$ o total de sequências de $n+1$ termos que satisfaz as condições dadas do problema. Dada uma sequência de $n$ termos, sempre é possível gerar uma sequência de $n+1$ termos adicionando o algarismo 1 ou 2 no início da sequência. Com isso, pelo princípio multiplicativo, é possível gerar $2 \cdot S_{n}$ sequências.

Suponha agora que as sequências de $n+1$ termos são iniciadas com o algarismo 0 . Como o problema não permite que se tenha dois algarismos 0 em sequência, o seguinte deve ser 1 ou 2 , seguido de $n-1$ algarismos. E, pelo princípio multiplicativo, podem ser formadas $2 \cdot S_{n-1}$ sequências.

Por fim, utilizando o princípio aditivo, modelamos o problema pela relação de recorrência,

$$
S_{n+1}=2 S_{n}+2 S_{n-1} .
$$

Para resolver essa relação de recorrência, que é de ordem 2, precisam-se determinar duas condições de contorno. Para isso, basta resolver os casos $S_{1}$ e $S_{2}$, que são facilmente encontrados ao se montar seus espaços amostrais.

Ao se utilizar um termo, as sequências que satisfazem o enunciado são (0), (1), (2), ou seja, $S_{1}=3$. Já para as sequências de dois termos, o espaço amostral é dado por (01), (02), (10), (11), (12), (20), (21), (22), ou seja, $S_{2}=8$.

Com isso, é possível resolver utilizando os métodos apresentados previamente.

\section{a) Método tradicional:}

O polinômio característico da expressão acima será dado por $p(z)=z^{2}-2 z-2$ que apresenta como raízes $\lambda_{1}=1-\sqrt{3}$ e $\lambda_{2}=1+\sqrt{3}$ e respectivas multiplicidades $m_{1}=1$ e $m_{2}=1$. Note que para $n=0$ o problema não faz sentido, com isso vamos alterar a fórmula (3) de forma que possam ser utilizados $S_{1}$ e $S_{2}$ como condição de contorno. Assim, a equação é dada por

$$
S_{n}=\beta_{1,1} \lambda_{1}^{n}+\beta_{2,1} \lambda_{2}^{n},
$$

faltando determinar as constantes $\beta_{1,1}$ e $\beta_{2,1}$.

Resolvendo o sistema linear,

$$
\left\{\begin{array}{c}
\lambda_{1} \beta_{1,1}+\lambda_{2} \beta_{2,1}=1 \\
\lambda_{1}^{2} \beta_{1,1}+\lambda_{2}^{2} \beta_{2,1}=1
\end{array}\right.
$$

obtêm-se $\beta_{1,1}=\frac{3-2 \sqrt{3}}{6}$ e $\beta_{2,1}=\frac{3+2 \sqrt{3}}{6}$.

Com isso, a solução da recorrência é dada por,

$$
S_{n}=\left(\frac{3-2 \sqrt{3}}{6}\right)(1-\sqrt{3})^{n}+\left(\frac{3+2 \sqrt{3}}{6}\right)(1+\sqrt{3})^{n} .
$$




\section{b) Método BenTaher-Rachidi:}

Utilizando o mesmo polinômio característico que o apresentado no item $a$ ), derivando $p$ obtemos o polinômio $p^{\prime}(z)=2 z-2$ e podemos calcular $p^{\prime}\left(\lambda_{1}\right)$ e $p^{\prime}\left(\lambda_{2}\right)$.

Utilizando as condições de contorno $V_{0}=S_{1}=3$ e $V_{1}=S_{2}=8$ para calcular $A_{1}=a_{1} V_{1} \mathrm{e}$ $A_{0}=a_{1} V_{0}+a_{0} V_{1}$, onde $a_{0}=2$ e $a_{1}=2$, são dados pelos coeficientes da relação de recorrência:

$$
\begin{gathered}
A_{0}=a_{1} V_{0}+a_{0} V_{1} \Rightarrow A_{0}=2 \cdot 3+2 \cdot 8 \Rightarrow A_{0}=22, \\
A_{1}=a_{1} V_{1} \Rightarrow A_{1}=2 \cdot 8 \Rightarrow A_{1}=16 .
\end{gathered}
$$

Com isso, utilizando a fórmula (5), a recorrência é dada por

$$
V_{n}=\frac{1}{p^{\prime}\left(\lambda_{1}\right)}\left(\frac{A_{0}}{\lambda_{1}^{0+1}}+\frac{A_{1}}{\lambda_{1}^{1+1}}\right) \lambda_{1}^{n}+\frac{1}{p^{\prime}\left(\lambda_{2}\right)}\left(\frac{A_{0}}{\lambda_{2}^{0+1}}+\frac{A_{1}}{\lambda_{2}^{1+1}}\right) \lambda_{2}^{n} .
$$

Substituindo os valores obtidos previamente e manipulando a equação, é possível escrever a recorrência como

$$
V_{n}=\left(\frac{9-5 \sqrt{3}}{6}\right)(1-\sqrt{3})^{n}+\left(\frac{9+5 \sqrt{3}}{6}\right)(1+\sqrt{3})^{n} .
$$

Note que a equação obtida utilizando a fórmula de BenTaher-Rachidi é válida para $n \geq 0$, enquanto a fórmula obtida no item $a$ ) só faz sentido para $n \geq 1$. Apesar das fórmulas aparentarem levemente diferentes, pode-se provar que $V_{n}=S_{n+1}$. De fato,

$$
\begin{gathered}
S_{n+1}=\left(\frac{3-2 \sqrt{3}}{6}\right)(1-\sqrt{3})^{n+1}+\left(\frac{3+2 \sqrt{3}}{6}\right)(1+\sqrt{3})^{n+1}, \\
\Rightarrow S_{n+1}=\left(\frac{3-2 \sqrt{3}}{6}\right)(1-\sqrt{3})(1-\sqrt{3})^{n}+\left(\frac{3+2 \sqrt{3}}{6}\right)(1+\sqrt{3})(1+\sqrt{3})^{n}, \\
\Rightarrow S_{n+1}=V_{n}=\left(\frac{9-5 \sqrt{3}}{6}\right)(1-\sqrt{3})^{n}+\left(\frac{9+5 \sqrt{3}}{6}\right)(1+\sqrt{3})^{n}, \\
\Rightarrow S_{n+1}=V_{n} .
\end{gathered}
$$

Logo, ambas são soluções da mesma recorrência, como esperado.

\section{Considerações finais}

Como visto, os dois métodos são simples de ser aplicados e podem ser utilizados para resolver problemas de recorrências lineares homogêneas de qualquer ordem superior com raízes simples. Apesar do método de BenTaher-Rachidi não ser ensinado em cursos de matemática, seu uso para solução de recorrências como as vistas é tão simples quanto o método tradicional, exigindo apenas 
conhecimento da derivada e outras operações básicas de polinômios, tornando-se uma alternativa para a resolução tradicional amplamente divulgada na literatura.

O mesmo comparativo pode ser descrito para os casos onde as raízes possuem multiplicidade. No entanto, o método BenTaher-Rachidi torna-se mais complexo, o que não o faz muito interessante senão manipulado computacionalmente. Somente um fato a ser discutido ainda é se, computacionalmente, o novo método BenTaher-Rachidi pode ser aplicado com resultados melhores que os já vistos na literatura. Utilizando exemplos clássicos, a resposta para essa pergunta parece não ser positiva, mas isso não retira o fato de o novo método ser uma alternativa interessante aos cálculos clássicos.

\section{Referências}

[1] Alves, F. R. V. "Sequência Generalizada de Fibonacci e Relações com o Número Áureo". Boletim Cearense de Educação e História da Matemática, v. 2, nº 6, p. 30-36, 2015.

[2] Carvalho, C. P. C., Morgado, A. C. O. Matemática Discreta. Sociedade Brasileira de Matemática, Brasil, 2015.

[3] Pinho, A. O problema dos coelhos. https://sites.google.com/site/leonardofibonacci7/oproblema-dos-coelhos, acesso em 28/04/2019.

[4] Taher, R. B., Rachidi, M. "Solving some generalized Vandermonde systems and inverse of their associate matrices via new approaches for the Binet formula". Applied Mathematics and Computation Volume 290, 1 November 2016, Pages 267-280 , v. 290, p. 267-280, 2016.

[5] Wolfram, S. A New Kind of Science.Wolfram Media,Champaign, IL, 2002. https:www.wolframscience.com, acesso em 24/04/2019.

Lucas S. C. de Sá

Universidade Federal de Mato Grosso do Sul Mestrado Profissional em Matemática em Rede Nacional $<$ lucas.zth@gmail.com>

Elen V. P. Spreafico Universidade Federal de Mato Grosso do Sul Instituto de Matemática <elen.spreafico@ufms.br>

Recebido: $17 / 06 / 2019$

Publicado: $03 / 02 / 2020$ 